
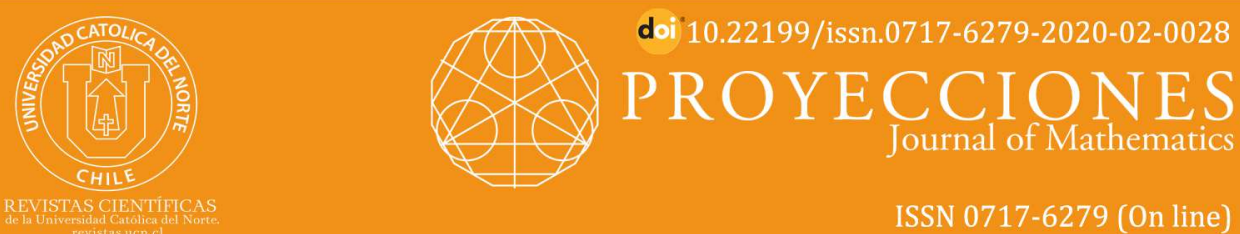

\title{
Branch duplication in trees: uniqueness of sedes and enumeration of sedes
}

Charles R. Johnson ${ }^{1}$

Jacob Lettie ${ }^{2}$

Sander Mack-Crane ${ }^{3}$

Alicja Szabelska-Beręsewicz ${ }^{4}$ (1) orcid.org/0000-0002-1806-0891

${ }^{1}$ The College of William and Mary, Dept. of Mathematics, Williamsburg, VA, U.S.A.

vrjohn@wm.edu

${ }^{2}$ Duke University, Dept.of Mathematics, Durham, NC, U.S.A.

$\square$ jrl47@duke.edu

${ }^{3}$ Case Western Reserve University, Dept of Mathematics, Applied Mathematics and Statistics, Cleveland, OH, U.S.A

vmack-crane@case.edu

${ }^{4}$ Poznan University of Life Sciences, Dept. of Mathematical and Statistical Methods, Poznan, Poland.

vaszabelska@gmail.com

Received: June 2019 | Accepted: November 2019

\section{Abstract:}

By the process of branch duplication, any tree may be generated from a type of tree called a seed. We describe the correspondence between trees and seeds by showing that each tree grows from a unique seed and giving an algorithm to produce this seed. Using results about the structure of seeds, we enumerate the seeds of a given diameter.

Keywords: Branch duplication in tres; Enumeration of sedes; Rooted tres; Seeds; Uniqueness of seeds.

MSC (2010): 05C30, 05C50, 65-04.

\section{Cite this article as (IEEE citation style):}

C. R. Johnson, J. Lettie, S. Mack-Crane, and A. Szabelska-Beręsewicz, "Branch duplication in trees: uniqueness of sedes and enumeration of sedes", Proyecciones (Antofagasta, On line), vol. 39, no. 2, pp. 451-465, Apr. 2020, doi: 10.22199/issn.0717-6279-2020-02-0028.

Article copyright: (C) 2020 Charles R. Johnson, Jacob Lettie, Sander Mack-Crane and Alicja Szabelska-Beręsewicz. This is an open access article distributed under the terms of the Creative Commons Licence, which permits unrestricted use and distribution provided the original author and source are credited. 


\section{Introduction}

Recently $[1,2]$, the notation of branch duplication in a tree has been introduced and become a key tool in determining the possible lists of multiplicietes for the eigenvalues of symmetric matrices, whose graph is a tree. It has long been known [3] that the diameter (measured in vertices) is a lower bound for the minimum number of distinct eigenvalues.

Here we investigate a class of trees called seeds, and an analogous class of rooted trees called rooted seeds. Seeds are the elemental trees with no repeated structure, i. e. no branches have been duplicated. All trees may be constructed from seeds via combinatorial branch duplication, so that seeds capture the most basic structure of a tree.

Definition 1. Given a tree $T$ and a branch $B$ emanating from vertex $v$, (combinatorial) branch duplication (of branch $B$ ) is the process of producing a new tree $T^{\prime}$ from $T$ by attaching a new branch identical to $B$ at $v$; we require that introducing this new branch does not increase the diameter of the tree. A seed is a tree that cannot be produced from another tree by branch duplication.

The requirement that diameter is not increased is an important feature of the application of seeds to inverse eigenvalue problems, which we review shortly. Rooted branch duplication and rooted seeds are defined analogously for rooted trees, with the requirement that a "branch duplication does not increase the diameter" replaced by the requirement that "the root node does not lie in the branch to be duplicated".

It will be shown (section 2) that each tree comes from a unique seed; that is, no tree exists that can be produced by branch duplication from two non-isomorphic seeds. Since this is the case, we may refer to the notion of a tree being in the family of the seed from which it was produced, so that families of distinct seeds do not intersect. Since all trees are in the family of some seed, and the families do not intersect, these families partition trees of a given diameter.

Example 2. The following tree is a seed, as at no vertex are there two isomorphic branches. 
We may duplicate one of this tree's branches in a way that does not increase the diameter of the tree to obtain a tree in the seed's family.

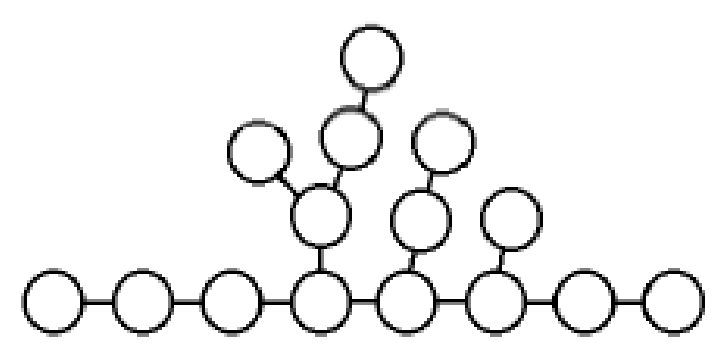

Figure 1: A seed of diameter 7 .

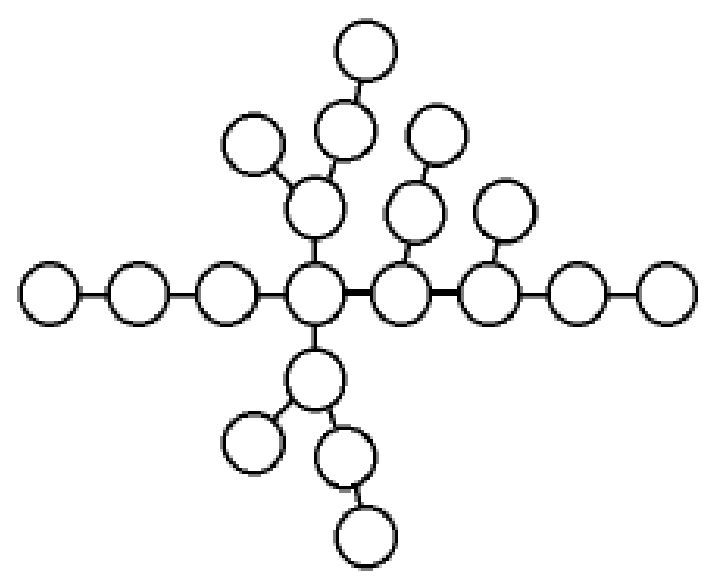

Figure 2: A tree produced from the previous tree by branch duplication.

Seeds and branch duplication were originally defined in [1] for the purpose of studying inverse eigenvalue problems. We will briefly describe this important application.

Given a labeled graph $G$ on $n$ vertices, an $n \times n$ real symmetric matrix $A$ is said to have graph $G$ if the off-diagonal entry $a_{i j}$ is nonzero precisely when $G$ has an edge between vertices $i$ and $j$ (diagonal entries are unrestricted). 
The inverse eigenvalue problem for a graph $G$ asks: what are the spectra of symmetric matrices with graph $G$ ? For this problem it is very useful to know the possible lists of multiplicities of eigenvalues for a symmetric matrix with graph $G$. A key element is the minimum number of distinct eigenvalues that such a matrix must have.

Seeds are applied to the problem in the following way. When a tree undergoes (combinatorial) branch duplication, it is possible to carry out a similar process on a matrix corresponding to that tree called algebraic branch duplication. The following theorem describes a key formula that gives the result of performing branch duplication on a given graph, and performing the corresponding algebraic branch duplication on its matrix.

Theorem 3 ([1], Theorem 1). Let $T$ be a tree and $A$ a matrix with graph $T$. Let $T^{\prime}$ be a tree obtained from $T$ by the (combinatorial) duplication of a branch $B$ whose vertex labels are the row and column indices of the principal submatrix $C$ of $A$; let $A^{\prime}$ be the matrix obtained from $A$ by the corresponding algebraic branch duplication. Then the matrix of $T^{\prime}$ is $A^{\prime}$ and $p_{A^{\prime}}(x)=p_{A}(x) p_{C}(x)$, in which $p_{M}(x)$ denotes the characteristic polynomial of matrix $M$.

If we are able to construct a matrix $A$ corresponding to a seed $S$ with the property that the eigenvalues of each branch (i.e. the eigenvalues of the principal submatrix corresponding to the branch, as in Theorem 3) are all eigenvalues of the whole matrix, then we may apply Theorem 3 to show that any tree that can be obtained from $S$ by branch duplication is the graph of a matrix with no more distinct eigenvalues than $A$.

Since the minimum number of distinct eigenvalues among matrices whose graph is a tree is bounded below by one plus the diameter of the tree [3], by carrying out the process described in the previous paragraph for all seeds of diameter less than 6 , it is shown in [1] that every tree of diameter less than 6 achieves this bound. (Note that $[1,2,3]$ define diameter in terms of vertices, which is convenient in the multiplicity literature. Here, we measure diameter in terms of edges, which is common in graph theory literature.)

More applications of seeds to inverse eigenvalue problems may be found in $[1,2]$.

For these applications and possible others it is important to understand the combinatorial aspects of seeds and branch duplication, and this is our present purpose. In Section 2 we discuss the correspondence between trees and seeds. In particular, we show that every tree has exactly one seed, 
from which it can be produced by branch duplication. In section 3, we give an algorithm that determines the seed of a given tree. In Section 4 we enumerate the rooted seeds of a given height, and then use this result to enumerate the seeds of a given diameter. Though it begins slowly, the number of seeds of a given diameter eventually grows very rapidly

For clarity, we now record the basic definitions necessary for this paper.

By a tree we mean a simple undirected graph with no cycles and only finitely many vertices. We will always use the modifier rooted when speaking of rooted trees and seeds, i.e. trees with a distinguished vertex called the root.

A branch of a tree is a subtree whose deletion leaves the resulting graph connected. A branch may be defined by identifying a vertex and a direction from that vertex, i. e. a neighbor.

The diameter of a tree is the number of edges in a longest path. Similarly, the height of a rooted tree is the number of edges in a longest path beginning at the root.

\section{Seeds and Their Uniqueness for a Tree}

In this section we show that every tree has a unique seed.

To prove that every tree has a unique seed it is useful to introduce coloring. Consider the following process: begin with a seed whose vertices are colored white, and perform a branch duplication, but color the vertices of the new branch black. Repeat as desired. The result is a tree with black and white vertices, whose subtree of white vertices is the seed from which the tree was produced. Any tree produced in this way we call a colored tree. For any tree $T$, there is at least one colored tree with underlying graph $T$ (by underlying graph we mean the same graph with coloring forgotten). For ease of bookkeeping we denote colored trees and branches in the same way as their underlying graphs, with a tilde added.

We also find it useful to recognize duplicates. A duplicate branch of a tree $T$ is a branch $B$ such that upon deleting $B$ to obtain a tree $T^{\prime}$, the trees $T$ and $T^{\prime}$ have the same diameter and $T$ may be produced from $T^{\prime}$ by a single branch duplication. Note that in this case there is a duplicate pair of identical branches $B, B^{\prime}$ emanating from the same vertex of $T$, and there is an automorphism of $T$ which sends vertices of $B$ to vertices of $B^{\prime}$, vertices of $B^{\prime}$ to vertices of $B$, and fixes all other vertices.

There may be more than two identical branches attached to a vertex, i.e. a branch may belong to more than one set of duplicate pairs. In this case 
it is convenient to recognize the full duplicate set of all identical branches attached to that vertex. A minimal duplicate branch is a duplicate branch which does not contain any duplicate branches, and we define similarly a minimal duplicate pair.

Any tree can be obtained from a seed by a sequence of branch duplications, and the length of such a sequence (i.e. number of branch duplications) for a particular tree is bounded e.g. by the number of vertices of the tree. Define the duplicate branch number of a tree (or colored tree) to be the length of a longest sequence of branch duplications that produces the tree from a seed. A tree is a seed exactly when it has duplicate branch number 0 .

To prove that every tree has a unique seed, we prove that colored trees whose underlying graphs are isomorphic must have isomorphic white subtrees; this shows that the seed depends only on the underlying graph, not the sequence of branch duplications used to produce it. We use induction on duplicate branch number, and the following lemma ensures that we may apply the induciton hypothesis after deleting an appropriate branch.

Lemma 1. Let $\tilde{T}$ be a colored tree, and $\tilde{T}^{\prime}$ a tree obtained by deleting a black minimal duplicate branch $\tilde{B}$ from $\tilde{T}$. Then $\tilde{T}^{\prime}$ is a colored tree with duplicate branch number strictly less than $\tilde{T}$.

Proof. To show $\tilde{T}^{\prime}$ is a colored tree we must show that it can be produced from its white subtree by branch duplication.

A sequence of branch duplications may always be modified (without changing the resulting tree) so that each duplication creates a minimal duplicate pair. First, if a branch of a seed contains a duplicate pair, then it must be that deleting one of the duplicates would decrease the diameter of the seed; but then duplicating the whole branch would increase the diameter, since the diameter includes subpaths of the two duplicates. That is why every branch duplication from a seed must produce a minimal duplicate pair. Also, if a branch is duplicated and then a branch containing those duplicates is duplicated, we may instead duplicate the larger branch first and then in each of the resulting branches duplicate the sub-branch. By repeatedly performing such replacements we obtain a sequence of branch duplications producing $\tilde{T}$ from its seed such that every duplication produces a minimal pair. Note that these replacements do not affect the resulting colored tree.

Now since $\tilde{B}$ is minimal and black it must be that $\tilde{B}$ was the result of a single branch duplication. To produce $\tilde{T}^{\prime}$ from its white subtree we may 
use the same sequence that produces $\tilde{T}$, leaving off the duplication that produces $\tilde{B}$.

Also, by taking a longest sequence of branch duplications by which $\tilde{T}^{\prime}$ can be produced, and extending by one branch duplication to produce $\tilde{T}$, we see that the duplicate branch number of $\tilde{T}$ must be strictly greater than the duplicate branch number of $\tilde{T}^{\prime}$.

The next lemma is used to find an appropriate branch to delete before applying the induction hypothesis.

Lemma 2. If a colored tree $\tilde{T}$ which is not a seed has a full set of duplicate branches colored white, then there is a pair of duplicate branches with strictly fewer vertices.

Proof. If $\tilde{T}^{\prime}$ has a full set of duplicate branches colored white, then there must be exactly two such branches, for the white subtree (being a seed) cannot contain three copies of a branch: any diameter of the seed may touch at most two of the branches, so deleting the third cannot decrease its diameter. Call these duplicate branches $\tilde{B}, \tilde{B}^{\prime}$, and their shared parent vertex $v$.

Since $\tilde{B}$ and $\tilde{B}^{\prime}$ are not duplicates in the seed, despite being identical branches on the same vertex, it must be that deleting one of them reduces the diameter of the seed. Thus every diameter of the seed passes through $\tilde{B}$ and $\tilde{B}^{\prime}$ (and no other branch at $v$ ). However, $\tilde{B}$ and $\tilde{B}^{\prime}$ are duplicates in $\tilde{T}$, so there is a diameter that does not pass through one of them.

No branch of the seed containing $v$ can be duplicated, for this would increase the diameter of the tree. Thus any branch duplication in the sequence used to produce $\tilde{T}$ must duplicate a branch at $v$ or a sub-branch of a branch at $v$. A new diameter cannot have been created by duplicating a branch at $v$ other than $\tilde{B}$ or $\tilde{B}^{\prime}$; if it was, then before the duplication there must have been a diameter passing through this branch. For the same reason a new diameter also cannot have been created by duplicating a sub-branch of a branch at $v$.

It must be that in producing $\tilde{T}$ from its white subtree one of $\tilde{B}, \tilde{B}^{\prime}$ was duplicated; call the duplicate $\tilde{B}^{\prime \prime}$. By hypothesis $\tilde{B}$ and $\tilde{B}^{\prime}$ form a full duplicate set, so $\tilde{B}^{\prime \prime}$ is not a branch of $\tilde{T}$, and the only way this can happen is if a proper sub-branch of $\tilde{B}^{\prime \prime}$ was duplicated. This creates a duplicate pair with strictly fewer vertices than $\tilde{B}, \tilde{B}^{\prime}$. If these branches are a duplicate pair in $\tilde{T}$, then we are done; if not, then it must be that a proper sub-branch of one of them was duplicated, which creates a duplicate pair 
with still fewer vertices. Repeating, we must eventually find the desired duplicate pair.

We are now prepared to prove the main theorem of this section.

Theorem 3. Every tree has a unique seed from which it can be produced by branch duplication.

Proof. Let $\tilde{T}, \tilde{T}^{\prime}$ be any two colored trees with isomorphic underlying graphs $T, T^{\prime}$; we seek to show that $\tilde{T}, \tilde{T}^{\prime}$ have isomorphic white subtrees. We proceed by induction on the duplicate branch number of $T \simeq T^{\prime}$.

Suppose the duplicate branch number of $T \simeq T^{\prime}$ is 0 , i.e. they are seeds. Then $\tilde{T}, \tilde{T}^{\prime}$ must be completely white, and any isomorphism between $T, T^{\prime}$ gives an isomorphism of the white subtrees of $\tilde{T}, \tilde{T}^{\prime}$.

Now suppose the duplicate branch number of $T \simeq T^{\prime}$ is greater than 0 , and suppose the claim (that colored trees with isomorphic underlying graphs have isomorphic white subtrees) is true for colored trees of smaller duplicate branch number. Choose a full set of duplicate branches in $T$ which have the fewest vertices (per branch) of any set of duplicate branches, and note that these are minimal duplicate branches; call them $B_{1}, \ldots, B_{k}$ and their colored analogues $\tilde{B}_{1}, \ldots, \tilde{B}_{k}$. By choosing an isomorphism $T \rightarrow T^{\prime}$ we obtain a matching set of duplicates $B_{1}^{\prime}, \ldots, B_{k}^{\prime}$ in $T^{\prime}$ and $\tilde{B}_{1}^{\prime}, \ldots, \tilde{B}_{k}^{\prime}$ in $\tilde{T}^{\prime}$.

Since $\tilde{B}_{1}, \ldots, \tilde{B}_{k}$ contain no duplicate branches each must be all white or all black; also not all of them can be white for this would contradict Lemma 5 . The same argument applies to $\tilde{B}_{1}^{\prime}, \ldots, \tilde{B}_{k}^{\prime}$. Without loss of generality $\tilde{B}_{1}, \tilde{B}_{1}^{\prime}$ are black, for we may reorder the indices to make $\tilde{B}_{1}$ black and choose an appropriate isomorphism to make $\tilde{B}_{1}^{\prime}$ black.

By Lemma 4 deleting $\tilde{B}_{1}, \tilde{B}_{1}^{\prime}$ from $\tilde{T}, \tilde{T}^{\prime}$ results in a pair of colored trees with duplicate branch number strictly less than that of $\tilde{T}, \tilde{T}^{\prime}$. Also, the pair have isomorphic underlying graphs, since there is an isomorphism $T \rightarrow T^{\prime}$ that identifies $B_{1}$ with $B_{1}^{\prime}$. Thus we may apply the induction hypothesis to see that the pair have isomorphic white subtrees. But since the deleted branches $\tilde{B}_{1}, \tilde{B}_{1}^{\prime}$ had no white vertices, it must be the case that $\tilde{T}, \tilde{T}^{\prime}$ have isomorphic white subtrees. This completes the induction.

Since any seed from which a tree can be produced is realized as the white subtree of some colored tree with that underlying graph, it follows that any tree can only be produced from a single seed.

A directly analogous proof gives Theorem 6 in the case that branch duplication is allowed to increase the diameter of the tree; in fact, Lemma 
5 is no longer needed in this case, as duplicate branches can never be all white if deleting branches may reduce the diameter.

\section{Algorithm that Determines the Seed of a Tree}

We know that each tree comes from a unique seed with given diameter. We would like to have an algorithm that enables us to produce the seed for a given tree. There are two main approaches for trimming a tree. The first involves looking for the biggest branches that are duplicated, trimming them, and repeating the search for the biggest branches. In the other approach the focus is put on the smallest branches. If the tree contains a small number of big duplicated branches the first algorithm would be preferable. However in the general case this approach would involve rechecking bigger branches after trimming smaller ones. In the second approach we need to check each vertex only once. The algorithm described here is based on the second approach.

Note that trees of odd diameter may have many longest paths that achieve the tree's diameter, but they all must share a single edge at the midpoint of this path. We will call this edge the central edge of the tree. Trees of even diameter may also have many longest paths, and all of these paths must share a central vertex, which we will call the central vertex of the tree.

For a tree with even diameter let us consider a rooted tree, for which the central vertex is declared as a root. In the case of tree with odd diameter let us consider two trees that are the result of removal of central edge and the two vertices of this edge declared as roots in obtained trees. In both cases each vertex can be labeled based on length of the shortest path to the root. For trees with odd diameter the same colors are used for labeling in both trees. Thus if the diameter is denoted as $d$, we have $n$ colors to choose with $d=2 n$ for even diameter and $d=2 n+1$ for odd diameter. At each step of the algorithm the duplicated branches of vertices of a particular color are trimmed, starting from the deletion of duplicated pendent vertices. At each next step we focus on branches of a new particular height, that is sequentially increasing, since all the duplicated branches of smaller height were trimmed at the previous steps of the algorithm. The final step considers the branches from the root. It differs for trees with odd and even diameter. The algorithm can be described in detail as follows:

1. Let $\mathrm{k}$ denotes the length of a path to the root. Set $k=n-1$. 
2. Let $V_{r}$ denotes the root of tree or the two roots in subtrees for tree with even diameter and odd diameter, respectively. Search for all the vertices at distance $k$ from $V_{r}$, i.e. $C_{k}=\left\{v: \min _{r} d\left(v, V_{r}\right)=k\right\}$. For each vertex $v$ in set $C_{k}$ check if any of the branches of $v$ are duplicated. Trim duplicates, that appear.

3. Check if $k=0$. If yes go to step (4). If no, set $k=k-1$ and go to step (2).

4. a) If the diameter is odd, $V_{r}$ consists of two vertices. For each of them check separately if any of its branches are duplicated. If yes, trim duplicates.

b) If diameter is even, $V_{r}$ consists of only one vertex. Count the number of branches of height $n$ attached to $V_{r}$. If all of these branches are duplications of the same branch, then leave two of them and trim the rest. If there are at least two different branches, then trim all the duplicates.

Note that reducing the number of branches of a tree with even diameter will not decrease the diameter, since for each vertex in the root, at least two branches of height $n$ will remain.

An example of usage of the algorithm can be found in Figure 3
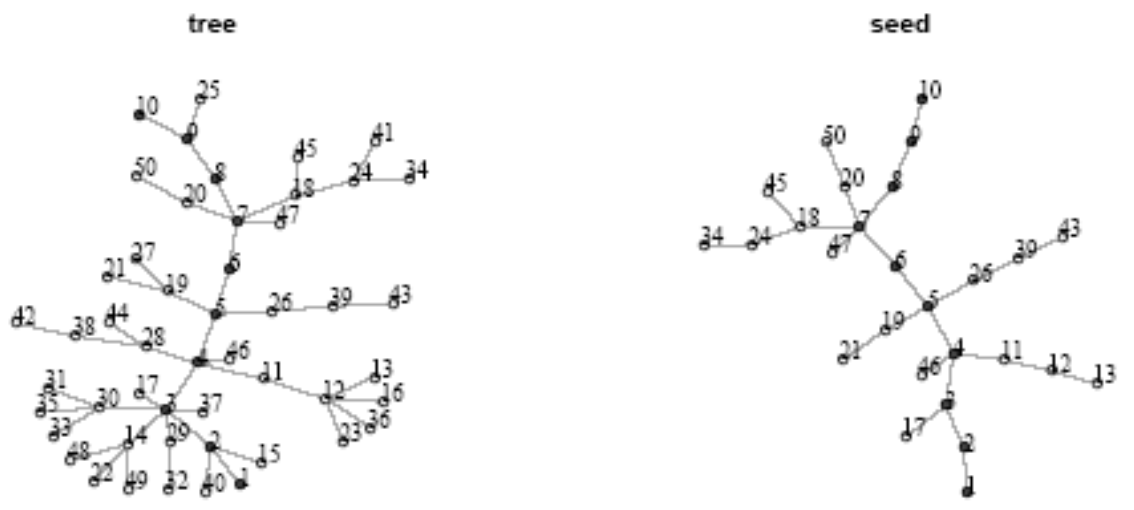

Figure 3: Result of the algorithm for 50 vertices and diameter 9. 
The computational complexity of the algorithm can be tested by simulations. For this purpose trees with given numbers of vertices (namely 200, 400, 600 vertices) and different diameter were generated using the $\mathrm{R}$ platform, version 3.1.3 [4]. For every tree produced the time of running the algorithm was measured. The results of the tests are presented in Figure 4 .

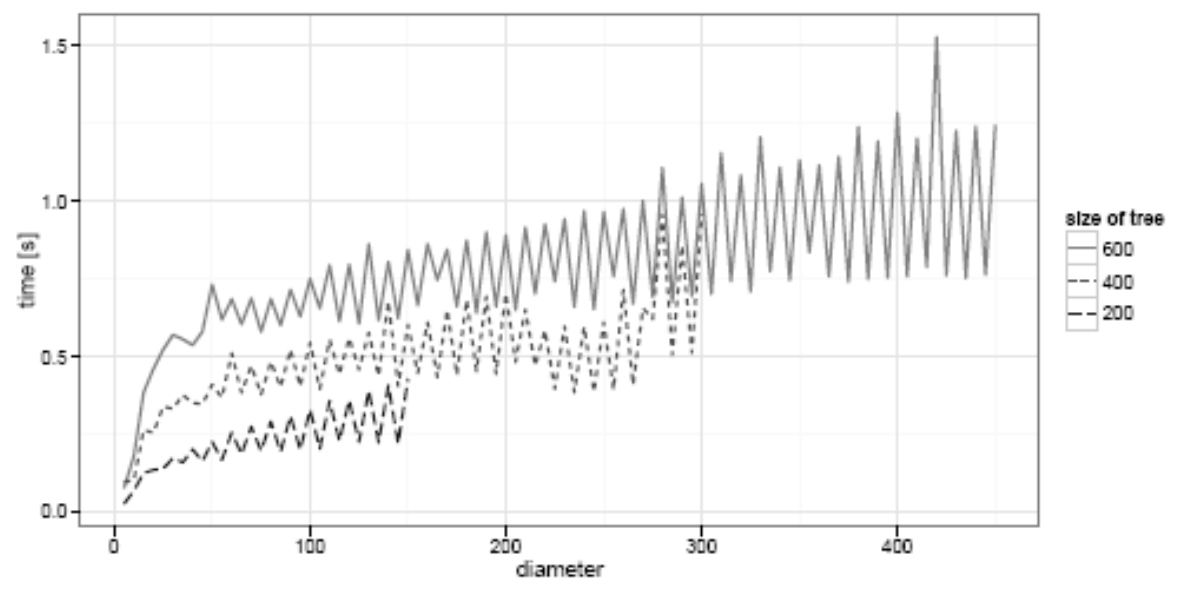

Figure 4: Computational complexity of the algorithm for trees with chosen number of vertices and diameter.

\section{Enumeration of Seeds of a Given Diameter}

Using some helpful facts about seeds, we are able to construct a function that returns the number of different seeds of a given diameter. To do this, we will show that unrooted seeds may be decomposed into a set of rooted seeds. Then we will enumerate the rooted seeds of a given height. The problem is then reduced to counting the number of decompositions.

\subsection{Breaking Down Seeds Using Rooted Seeds}

We use again the notations of "central edge" and "central vertex" of a tree.

Lemma 1. A tree of diameter $2 n+1$ is a seed if and only if the removal of its central edge and declaring the two vertices of this edge as roots in the two resulting tree, results in two rooted seeds. 
Proof. Since rooted seeds are free of branch duplication, two height $n$ rooted seeds can be attached by their roots, and the resulting tree will also be free of branch duplication. To see the reverse, notice that cutting a seed at its middle edge must result in two rooted seeds that do not feature rooted branch duplication, for if such a duplicate existed the original tree would not have been a seed.

Lemma 2. A tree of diameter $2 n$ is a seed if and only if the removal of its central vertex, then declaring all its neighbors as roots in their branches, results in either a forest of different rooted seeds with at least two rooted seeds of height $n-1$, or a forest of different rooted seeds of height less than $n-1$ together with exactly two identical rooted seeds of height $n-1$.

Proof. Since rooted seeds are free of branch duplication, any set of unique rooted seeds of height $\leq n-1$ may be added to the central vertex to create a seed, so long as at least 2 height $n-1$ rooted seeds are added to ensure the desired diameter. If no more than 2 height $n-1$ rooted seeds are added, those two seeds are permitted to be identical; they cannot be the result of branch duplication because the removal of either would decrease the diameter of the tree. This process must result in the creation of a seed because the rooted seeds themselves are free of branch duplication, and the restrictions on their uniqueness prevent them from being duplicates of each other.

For the converse, removal of the central vertex must result in rooted seeds, since the seed itself has no occurrences of branch duplication. These rooted seeds must be unique to avoid branch duplication, with the one exception being the possibility of the only two height $n-1$ rooted seeds being identical. In either case, there must be at least two components of height $n-1$, since the diameter of the original seed was $2 n$.

Since all odd diameter trees have a unique central edge, and all even ones have a unique central vertex, a set of rooted seeds conforming to the conditions of the above lemmas must produce a seed once connected by the central component of the tree. Any such "gluing" of rooted seeds is unique; seeds can be characterized precisely by the collection of rooted seeds connected to their central vertex or edge. Thus, counting seeds of diameter $n$ simply amounts to counting the number of valid sets of rooted constituent seeds. 


\subsection{The Number of Rooted Seeds}

Since seeds are just rooted seeds glued together, it will be helpful to count the number of rooted seeds of a given height. Fortunately, a lemma can reduce counting rooted seeds to a decomposition problem similar to that for unrooted seeds.

Lemma 3. A rooted tree of height $k$ is a rooted seed if and only if the removal of its root, together with the marking of each of its neighbors as a root in its own branch, results in a forest of distinct rooted seeds, among which the maximum height is $k-1$.

Proof. Since at no vertex of a rooted seeds do duplicate branches occur, a collection of distinct rooted seeds, whose maximum height is $k-1$ may be attached at their roots to a designated new vertex to form a new rooted seed of height $\mathrm{k}$, when the new vertex is designated as the root in a new tree.

For the converse, removal of the root from the rooted seed must a) leave only rooted seeds, since there are no duplicated branches at any vertex of the seed, and b) the resulting forest of rooted trees can include no duplicates, else the original rooted seed would have been the result of branch duplication in a smaller tree. Removal of the root must decrease the height of the remaining rooted trees, with any one in whose direction the height $\mathrm{k}$ was attained now being height $\mathrm{k}-1$.

Thus, a set of rooted seeds conforming to the conditions of the above lemma must produce a new seed when attached by their former roots to a new vertex designated as a root. Any such "gluing" of rooted seeds to form a new one is unique, so that rooted seeds are characterized by the list of smaller rooted seeds attached to their root. Thus, the number of rooted seeds of height $k$ is precisely the number of sets of different rooted seeds, among which the maximum height is $k-1$. We will call such a set the component set of a given rooted seed.

We may now give a recursive formula for $a_{n}$, the number of rooted seeds of height $\leq n$. Clearly $a_{0}=1$, for the only rooted tree of height 0 is a lone root. To find $a_{n}$, we just have to count the number of component sets of height $<n$. Since there are $a_{n-1}$ rooted seeds of height $<n, a_{n}$ is simply the number of ways of choosing a subset of a set of cardinality $a_{n-1}$ things. Thus, $a_{n}=2^{a_{n-1}}$. Recall that the notation 


$$
a b=\underbrace{a^{a^{a}}}_{b \text { times }}
$$

represents $a$ being raised to its own power $b$ times. Since each term is 2 to the power of the previous term, we have $a_{n}=2 n$.

Thus, writing $r_{n}$ for the number of rooted seeds of height $n$, we see that

$$
r_{n}=a_{n}-a_{n-1}=2 n-2(n-1) .
$$

\subsection{The Number of Seeds}

Now that we know the number of rooted seeds of height $n$, we can count the number $s_{n}$ of seeds of diameter $n$ in a similar manner. Since any seed of diameter $2 n+1$ is just two rooted seeds of height $n$ connected by an edge joining their roots, $s_{2 n+1}$ is simply the number of ways of pairing any two such rooted seeds together. Repeats are allowed, so we find that

$$
\begin{gathered}
s_{2 n+1}=\left(\begin{array}{c}
r_{n} \\
2
\end{array}\right)+r_{n}=\frac{\left(r_{n}\right)\left(r_{n}+1\right)}{2} \\
=\frac{[(2 n)-(2(n-1))][(2 n)-(2(n-1))+1]}{2} .
\end{gathered}
$$

Counting the number of seeds of diameter $2 n$ is similar to counting the number of rooted seeds of height $n$. In fact, the two are the same. To count the number $b_{n}$ of seeds of even diameter no more than $2 n$, all we need to do is count the number of valid sets of rooted seeds that compose a seed of even diameter $\leq 2 n$. This is simply the number of collections of different rooted seeds of height no more than $n-1$, minus the number of such sets in which there is exactly one rooted seed of height $n-1$, plus the number of sets that are different except for their containment of exactly two rooted seeds of height $n-1$ that are identical. However, the number of sets that is being subtracted from the set of different rooted seeds is the number of sets that are being added to it, since the sets in the second category are simply those in the first category with a copy of the unique rooted seed of height $n-1$ added a second time. Thus, the number of seeds of even diameter no more than $2 n$ is simply the number of collections of different rooted seeds of height $\leq n-1$, and

$$
b_{n}=2^{a_{n-1}}=2^{2(n-1)}=2 n .
$$


So the number of seeds of diameter exactly $2 n$ is

$$
s_{2 n}=b_{n}-b_{n-1}=(2 n)-(2(n-1)) .
$$

Thus, the function

$$
\begin{gathered}
s_{2 n}=(2 n)-(2(n-1)) \\
s_{2 n+1}=\frac{[(2 n)-(2(n-1))][(2 n)-(2(n-1))+1]}{2}, n \geq 1 \\
s_{1}=1
\end{gathered}
$$

gives the number of seeds of a given diameter.

\section{Acknowledgements}

This work was supported by NSF grant DMS 0751964.

\section{References}

[1] C. R. Johnson and C. M. Saiago, "Branch duplication for the construction of multiple eigenvalues in an Hermitian matrix whose graph is a tree", Linear and multilinear algebra, vol. 56, no. 4, pp. 357-380, 2008, doi: $10.1080 / 03081080600597668$.

[2] C. R. Johnson and C. M. Saiago, "Diameter minimal trees", Linear and multilinear algebra, vol. 64, no. 3, pp. 557-571, 2015, doi: 10.1080/03081087.2015.1057097.

[3] A. Leal-Duarte and C. R. Johnson, "On the minimum number of distinct eigenvalues for a symmetric matrix whose graph is a given tree", Mathematical inequalities \& applications, vol. 5, no. 2, pp. 175-180, 2002, doi: 10.7153/mia-05-19.

[4] The R Fundation, "The R Project for Statistical Computing", R-project, 2016. [Online]. Available: https://www.r-project.org/ 